\title{
Small Molecules Induce Mesocrystal Formation: Nanoparticle Aggregation Directed by Self-Assembling Calixarenes
}

\author{
Andrew Baynton ${ }^{\mathrm{a}}$, Tomoko Radomirovic ${ }^{\mathrm{a}}$, Mark I. Ogden ${ }^{\mathrm{a}}$, Colin L. Raston ${ }^{\mathrm{b}}$, William R. Richmond ${ }^{\mathrm{a}}$, and \\ Franca Jones*a
}

\author{
${ }_{5}$ Received (in $\left.X X X, X X X\right)$ Xth $X X X X X X X X X 200 X$, Accepted Xth $X X X X X X X X X 200 X$ \\ First published on the web Xth $X X X X X X X X X 200 X$ \\ DOI: $10.1039 / \mathbf{b 0 0 0 0 0 0 x}$
}

\begin{abstract}
Calixarenes have been shown to induce mesocrystal formation of barium sulfate, despite being relatively low molecular weight 10 additives. Scanning probe microscopy has shown that a possible mechanism is the self-assembling properties of the calixarene resulting in steric stabilization of the nanoparticles, comparable to that typically requiring polymeric additives. Supporting this hypothesis, the calixarenes are effective at concentration of $\sim 0.01$ $15 \mathrm{mM}$, which is significantly lower than other low molecular weight additives known to induce mesocrystal formation, usually via charge stabilisation (zeta potential measurements excluded the charge stabilisation mechanism in the present examples).
\end{abstract}

Non-classical crystallization involves the assembly of 20 nanoparticles with sufficient order that the resulting mesocrystal is virtually indistinguishable from a single crystal using standard techniques such as powder XRD. ${ }^{1}$ This phenomena is regarded as being significant in better understanding biomineralisation processes, where proteins exhibit exquisite control of crystal ${ }_{25}$ growth. ${ }^{2,3}$ Synthetic polyelectrolytes, typically block copolymers, have been reported to induce the formation of mesocrystals in a wide range of inorganic systems, with extension to an organic material recently reported. ${ }^{4,5}$ The impact of a random copolymer, upon the crystallization of calcium carbonate has been studied in 30 detail, with the realisation that single crystals are virtually absent under all of the conditions studied, with non-classical crystallization dominating the system. ${ }^{6}$ These results highlight the significance of nanoparticle-mediated crystallization, and suggest that this mechanism of crystal growth may be widespread. An 35 important aspect of the mechanism of formation of mesocrystals is the stabilisation of the nanoparticles so that they have time to aggregate in a non-random fashion. Recent literature highlights that this stabilisation can occur in many different ways, through emulsions/micelle formation, magnetism, and charge stabilisation

40 just to name a few. ${ }^{7}$ In fact, for small molecules, the stabilisation mechanism is often simply assumed to be charge stabilisation without further investigation. ${ }^{7 \mathrm{~d}}$

Previously we reported on the use of the calix[4]arene framework, which usually exists in the cone conformation, to 45 control the orientation of moieties known to be significant in modifying crystal growth. Functionalizing the lower (phenolic) rim of the calixarene with aspartic acid, for example, produced a potent growth inhibitor. ${ }^{8}$ In the present study we focus on functionalizing the divergent upper rim with water solubilising 50 sulfonic acid/sulfonate or phosphonic acid /phosphonate groups, as molecules $\mathbf{1}$ and $\mathbf{2}$ respectively and assess the impact on the crystal growth of barium sulfate (Scheme 1). Barium sulfate has been shown to form mesocrystals, with their shape depending on parameters such as $\mathrm{pH}$ and the type of functional groups attached 55 to the di-block copolymer additive. ${ }^{5}$

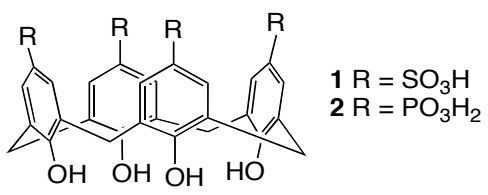

Scheme 1. Calixarene molecules investigated in this work

Based on the typical behaviour of sulfonate and phosphonate derivatives as crystal growth inhibitors, we expected $\mathbf{2}$ to have a ${ }_{60}$ greater inhibitory effect than $\mathbf{1}$, and this is indeed the case. The phosphonate, $\mathbf{2}$, is able to inhibit precipitation (involving barium chloride and sodium sulfate at a supersaturation ratio of $\sim 25$ ) completely over a 3 hour time period with as little as $0.013-0.014$ $\mathrm{mM}$, whereas even $0.2 \mathrm{mM}$ of 1 only inhibits barium sulfate ${ }_{65}$ precipitation by $34 \%$ relative to the control (supplementary information, Fig S2). Introduction of the additives also results in changes in crystal morphology (Fig $\mathbf{1}$ and supplementary information, Fig. S3). Both calixarene additives induce an initial change from the 'pillow' (Fig. 1A) to 'square tablet' like 70 particles, although again $\mathbf{2}$ is more potent than $\mathbf{1}$ (supplementary information, Fig. S3). As the concentration of calixarene is increased, rod-shaped particles are formed, which become rougher, smaller and flanged at the extremities with increasing concentrations of the additive. Remarkably, dumbbell shaped 75 particles eventually form, which can aggregate together.
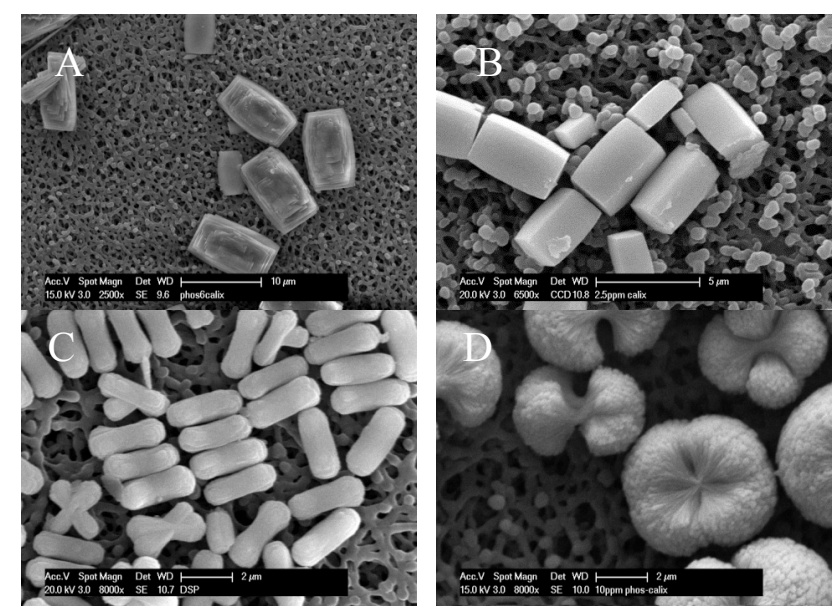

Figure 1. Barium sulfate particles formed in the presence of $p$ 80 phosphonatocalix[4]arene 2 at concentrations of A) $0.003 \mu \mathrm{M}$. B) $0.0034 \mathrm{mM} \mathrm{C}) 0.0067 \mathrm{mM}$ and D) $0.0134 \mathrm{mM}$ 
Larger (4L) batch experiments were performed to obtain solids for bulk characterization; in this case a fibre-like morphology was obtained (Fig. 2). Both particle shapes (dumbbell and fibre) are comparable to those observed for mesocrystals formed in the 5 presence of di-block co-polymers. ${ }^{5}$ The formation of possible mesocrystals in this system is all the more remarkable when the concentration is considered; $\sim 0.01 \mathrm{mM}$ when $\mathbf{2}$ is present versus $\sim 0.1 \mathrm{mM}$ for the block co-polymers. ${ }^{5}$
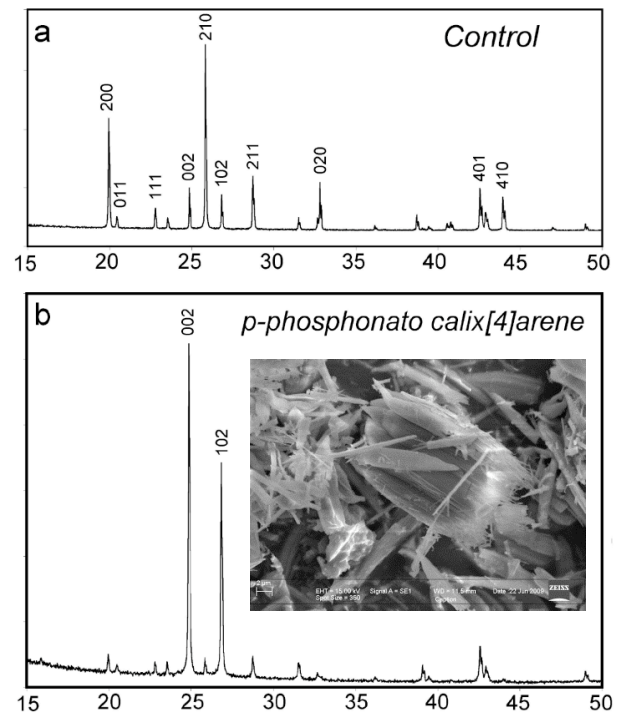

Figure 2. XRD patterns of barium sulfate particles formed on a $4 \mathrm{~L}$ batch scale, in the presence of (a) $0 \mathrm{mM}$ (control), and (b) $0.0134 \mathrm{mM} 2$.

The most striking differences between the control XRD pattern 15 and that with 2 present (Fig. 2) lies in the changes to relative intensities of specific peaks. The pattern of the product obtained in the presence of 2 reveals that the (002) and (102) reflections display intensities much greater than all other peaks in the pattern. This is characteristic of a strong preferred orientation 20 effect, consistent with the presence of particles with an anisotropic morphology. Line width analysis of multiple peaks in each pattern provided average crystallite sizes of $215 \mathrm{~nm}$ for the control sample and $160 \mathrm{~nm}$ for the material formed with 2 . Transmission electron microscopy (TEM) on ultramicrotomed ${ }_{25}$ samples was therefore used to determine the faces present (Fig 3).
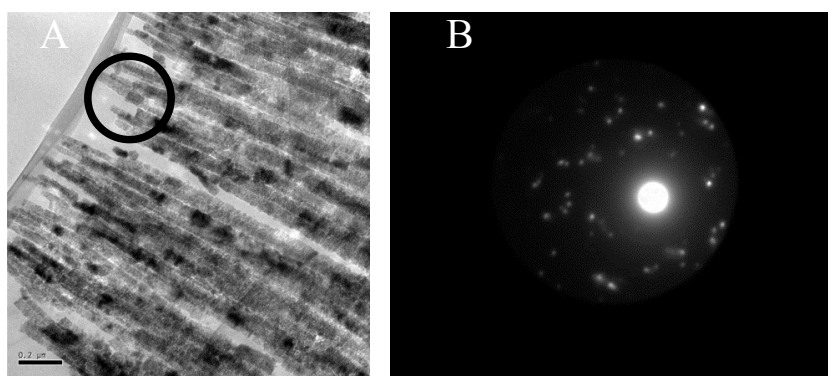

Figure 3. Barium sulfate particles formed in the presence of (A) 0.0134 mM 2 (4 L batch), and (B) SAED of the area within the highlighted circle 30 in (A)

The thin sections of particles viewed under the TEM show conclusively that these fibres are made up of smaller particles, and their selected area electron diffraction (SAED) pattern show significant if imperfect alignment, thereby confirming 35 mesocrystal formation. In addition, the indexing of this zone as the (100) (see supplementary information, Fig. S5) leads to the conclusion that the fibres are elongated in the (001) direction. Presumably, as found in previous studies, this is due to selective adsorption onto specific faces. ${ }^{9}$ Given that calixarene molecules 40 can induce mesocrystal formation, it was necessary to consider how these relatively small molecules can stabilize the nanoparticles to the same extent as block copolymers. ${ }^{5}$ The calixarenes used in this work are not surfactants nor do these molecules phase separate or form structures at an oil-water or air-

45 water interface thus negating these as possible mechanisms for mesocrystal formation. Two possibilities appear likely, namely, charge stabilization or self-assembly of the organic moiety at the crystal surface such that it achieves steric stabilization of the particles comparable to that of polymeric additives.

${ }_{50}$ The zeta potential of the precipitated barium sulfate in the presence of $2(0.0134 \mathrm{mM})$ was measured at $-17 \mathrm{mV}$, which, while being slightly negative, it is still within the range of zeta potentials $( \pm 30 \mathrm{mV})$ where the system is expected to be unstable and would spontaneously coagulate. ${ }^{10}$ Thus, charge stabilization

55 is unlikely to be a factor in barite mesocrystal formation in the presence of 2. Stabilization of some kind must be operating in order to avoid random aggregative processes. We therefore investigated the possibility of the calixarene self-assembly hypothesis via atomic force microscopy (AFM).

${ }_{60}$ Atomic resolution imaging of the barium sulfate crystal grown in the presence of the sulfonated calixarene at $26.71 \mathrm{mM}$ (Fig. 4) showed similar spacings to that expected for pure barium sulfate, with the parameters for a unit cell being 9.0 and $5.5 \AA$ (compared to 8.91 and $5.47 \AA$ for pure barite).

65

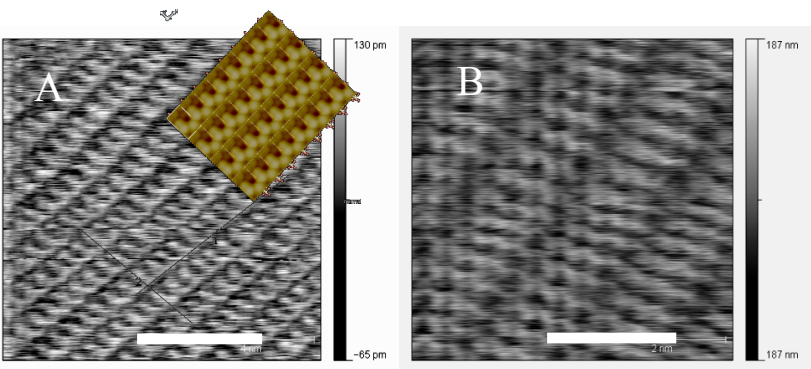

Figure 4. Atomic resolution AFM images of the barite crystal surface in the presence of (A) sulfonated calixarene 1 (simulated ${ }^{1}$ image for pure barium sulfate overlaid and (B) phosphonated 70 calixarene 2. (size bars represent 4 and $2 \mathrm{~nm}$ respectively)

In the case of the phosphonated calixarene, this is altered significantly in that the distance between the dark ridges is now $12.0 \AA$ while the other axis is $6.2 \AA$ apart. Thus, the surface imaged in the presence of the phosphonated calixarene does not 75 represent pure barium sulfate. The formation of self-assembled bi-layered structures is well established for $\mathbf{1}$, with the calixarenes in an alternate up-down arrangement with the sulfonate groups equally decorating the surfaces of the bi-layers. ${ }^{12}$ Recently, this type of arrangement has been established for $2,^{13}$ at different

\footnotetext{
${ }^{1}$ Simulation of the (001) barium sulfate surface using the isosurfaces functionality in GDIS ${ }^{[11]}$
} 
degrees of deprotonation of the phosphonic acid groups, with calcium counter ions interposed between bi-layers. ${ }^{14}$ Indeed, for the pristine phosphonic acid, association into nano-rafts prevails in solution, presumably with the same interlocking of the ${ }_{5}$ cavitands as in the bi-layers in the solid state, ${ }^{13,}{ }^{14}$ which is through hydrogen bonding and $\pi$-stacking. Such an arrangement of $\mathbf{2}$ on the surface of the nanoparticles is possible in the present study, in providing steric stabilization of the nanoparticles for controlled aggregation and formation of the mesocrysals, with the 10 potential for the calixarenes to bind to barium, like in the aforementioned calcium complex. ${ }^{14}$ Comparison of the structural data from these complexes shows the metal-free bilayers have unit cells $a=b=11.9381 \AA$ and $c=14.0678 \AA$. Thus, the AFM image is consistent with such a structure where the $c$ direction 15 would be normal to the image. However, two factors suggest this is not the case. Firstly, it is unlikely that a purely organic absorbed layer would be robust enough to be imaged in contact mode by AFM. Secondly, the simulated AFM images of the phosphonated bilayer are not consistent with the AFM image 20 obtained (see supplementary information, Fig. S5). It is most likely that the AFM image shows an ionic absorbed layer involving the calixarene in combination with barium and/or sulfate ions. The calcium complexes also involve bilayer formation and have lattice parameters that are comparable to the ${ }_{25} \mathrm{AFM}$ data with one structure having a $b$ axis of $\sim 12.85 \AA \AA^{13}$ The calcium complexes known to form with the phosphonated calixarene were taken and simulated atomic resolution AFM surfaces were constructed (see supplementary information, Fig. S6). These results show that a similar surface structure can be 30 seen particularly for the (001) face of form $2 .{ }^{13}$ Noting that we expect the parameters observed in the bulk structures would be somewhat modified for a surface absorbed structure we thus, propose that the AFM image is consistent with barium (and possibly sulfate) ions interacting with the phosphonated 35 calixarene in a bilayer type arrangement on the crystal surface, and it is this type of structure that sterically stabilises the nanoparticles during mesocrystal formation.

In summary, we note that these calixarenes are small molecules that are not surfactants and that the concentration of calixarene 40 required to induce mesocrystal formation is significantly lower than for other molecules studied thus far: for example, in the case of $\mathrm{EDTA}^{7 \mathrm{~d}} \sim 50 \mathrm{mM}$ is the concentration used. In this instance mesocrystal formation is observed with as little as $\sim 0.01 \mathrm{mM}$. These results demonstrate the broad potential for non-classical 45 crystallization induced by low molecular weight additives, and highlights that it should not be assumed that small molecules can only act by charge stabilising the nanoparticles prior to selfassembly. Given that small molecules of the type used in the present study can be readily and systematically modified, there is ${ }_{50}$ potential for using this approach in gaining control over the formation of mesocrystals, and this is of fundamental importance in ultimately developing their applications.

\section{Notes and references}

\section{Acknowledgements}

${ }_{55}$ We would like to thank John Murphy, Centre for Microscopy and Microanalysis (UWA) for ultramicrotomed samples, and the Australian Research Council for financial support.
${ }^{a}$ Curtin University of Technology, Department of Chemistry, GPO Box 60 U1987, Perth WA Australia 6845. Fax: 6189266 4699; Tel: 6189266 7677; E-mail: F.Jones@curtin.edu.au

b Centre for Strategic Nano-Fabrication, School of Biomedical, Biomolecular and Chemical Sciences, The University of Western Australia, Crawley, WA 6009, Australia

$\dagger$ Electronic Supplementary Information (ESI) available: [Experimental details, SEM images of barium sulfate formed in the presence of $\mathbf{1}$., SAED attribution to [100] zone, simulated AFM surfaces for calcium complexes of 2.].

[1] (a) M. Niederberger, H. Cölfen, Phys. Chem. Chem. Phys. 20068 3271-3287. (b) F. C. Meldrum, H. Cölfen, H. Chemical Reviews 2008108 4332-4432. (c) H. Cölfen, M Antonietti, Mesocrystals and Non Classical Crystallization, John Wiley \& Sons, Chichester UK. 2008.

[2] D. Gebauer, A. Verch, H. G. Borner, H. Cölfen, H., Crystal Growth and Design 20099 2398-2403.

[3] R. Q. Song, A. W. Xu, M. Antonietti, H. Cölfen, Angewandte Chemie Int. Ed. 2009 48 395-399.

80 [4] (a) L. Börger, H. Cölfen, M. Antonietti, M. Colloids and Surfaces A. 2000163 29-38. (b) H. Cölfen, M. Antonietti, Langmuir 1998 14582 (c) H. Cölfen, Current Opinion in Colloid and Interface Science 2003 8 23-31.

[5] L. Qi, H. Cölfen, M. Antonietti, Angew. Chem. Int. Ed. 200039 604-607. (b) L. Qi, H. Cölfen, M. Antonietti Chem. Mater. 200012 2392-2403.

[6] R. Q. Song, H. Cölfen, A. W. Xu, J. Hartmann, M. Antonietti, ACSNano, 20093 1966-1978.

[7] (a) M. Li, H. Schnablegger, S. Mann Nature 1999 402(6760) 393395 (b) A. Ahniyaz, Y. Sakamoto, L. Bergstrom, PNAS 2007104 17570-17574 (c) V. M. Yuwono, N. D. Burrows, J. A. Soltis, R. L. Penn J. Am. Chem. Soc. 2010 10.1021/ja909769a in press (d) I. C. Romero-Ibarra, G. Rodríguez-Gattorno, M. F. García-Sánchez, A. Sánchez-Solis, O. Manero Langmuir 2010 10.1021/la904197k in press.

[8] F. Jones, M. Mocerino, M. I. Ogden, A. Oliveira, G. M. Parkinson, Crystal Growth and Design 2005, 5, 2336-2343.

[9] (a) T. Wang, H. Cölfen Langmuir 200622 8975-8985 (b) T. Wang, A. Reinecke, H. Cölfen Langmuir 200622 8986-8994.

100 [10] T. Mäurer, B. Kraushaar-Czarnetzki, Helv. Chimica Acta 200184 2550-2556.

[11] S. D. Fleming, A. L. Rohl, Z. Kristallogr., 2005 220(5-6), 580584.

[12] L. Ling, Y. Alias, A. N. Sobolev, C. L. Raston Crystal Growth and Design 2009 94497-4503.

[13] T. E. Clark, M. Makha, A. N. Sobolev, H. Rohrs, J. L. Atwood, C. L. Raston Chem. Eur. J. 2008 14 3931-3938.

[14] A. D. Martin, A. N. Sobolev, M. A. Spackman, C. L. Raston, Crystal Growth \& Design 20099 3759-3764.

110 [15] S. Shinkai, K. Araki, T. Tsubaki, T. Arimura, O. Manabe J. Chem. Soc. - Perkins Trans. 1987 1(11) 2297-2299.

Supplementary Information:

\section{Experimental Section}


Calixarene molecules were synthesized in-house according to ( $\mathrm{p}$ sulfonatocalix[4]arene) ref. S1 and (p-phosphonatocalix[4]arene) ref. S2.

Samples were collected by filtration onto $0.22 \mu \mathrm{m}$ membranes. The solids were washed with ultrapure water and dried in a desiccator. 5 Subsequently, a portion of the filter paper was placed onto carbon-coated stub and stored in a desiccator. The samples were gold sputtered prior to viewing in a Philips XL30 scanning electron microscope. Larger, 4 L batches were prepared at ambient temperature and magnetically stirred for 3 hours prior to allowing the solids to settle overnight. The solids were 10 decanted, washed and filtered prior to drying in a dessicator. For TEM, the solids were fixed in resin and then ultramicrotomed into thin sections prior to viewing in a Jeol $2011 \mathrm{TEM}$ at $200 \mathrm{kV}$.

The solids collected from the large $4 \mathrm{~L}$ batch were analysed on a Bruker D8 Advance using $\mathrm{Cu} \mathrm{K}_{\mathrm{a}}$ radiation on a low background holder 15 spinning at $30 \mathrm{rpm}$. The 2 theta range was $15-50$ degrees with a step size of $0.001^{\circ}$ and a divergence slit of $0.3^{\circ}$. A LynxEye detector was used.

The zeta potential was measured using the Malvern NanoZeta ZS and a plastic zeta cell on particles in the solution they were formed in, at a phosphonated calixarene concentration of $0.013 \mathrm{mM}$.

20 AFM experiments were performed on a PicoPlus using a standard silicon nitride cantilever with a flow-through cell attachment. The same procedure was used for all samples. A freshly cleaved mineralogical barium sulfate sample was fixed to a metallic stub and the flow cell was flushed with filtered ultrapure water (resistivity $>18 \mathrm{M} \Omega \mathrm{cm}$ ) using a 25 precision, dual syringe pump run at $0.2 \mathrm{~mL} / \mathrm{min}$ and Gelman $0.2 \mu \mathrm{m}$ Supor $^{\circledR}$ membrane filters attached to the syringes. One syringe had the water replaced with barium chloride solution $(0.1 \mathrm{mM})$ and the other with sodium sulfate solution $(0.1 \mathrm{mM})$. This was then flushed through the cell at a rate of $0.2 \mathrm{~mL} / \mathrm{min}$ as per the water. Finally, the barium chloride

30 solution was replaced with a solution containing barium chloride plus the calixarene at a known concentration and the sodium sulfate solution was topped up as necessary. This was then flushed through the cell at a rate of $0.2 \mathrm{~mL} / \mathrm{min}$. For atomic resolution images, the pump was stopped and the system allowed to equilibrate while taking images.

35 Unseeded, de-supersaturation curves were obtained using a glass reactor kept at $25^{\circ} \mathrm{C}$ by a water bath and monitored using a conductivity probe (WTW LF 197 Conductivity meter). An overhead stirrer (150 rpm) kept the solids in suspension. The method of precipitation is the same as described in previous publications ${ }^{\mathrm{S} 3}, \mathrm{~S} 4, \mathrm{~S} 5$ and consisted of equilibrating $400.249 \mathrm{mM} \mathrm{BaCl}_{2}$ then adding $1 \mathrm{~mol}$ equivalent of $\mathrm{Na}_{2} \mathrm{SO}_{4}$ solution to initiate crystallization. The total volume for all experiments was $201 \mathrm{~mL}$. The linear region of the de-supersaturation curve (graph of conductivity versus time) was used to calculate the observed de-supersaturation rate. The $\mathrm{pH}$ for all experiments was 6.0 except where specified. The 45 calixarene was prepared as a stock solution of $1000 \mathrm{ppm}$ and the desired volume was added to the barium chloride solution prior to the addition of sulfate (keeping the total volume constant by adjusting the water addition). The de-supersaturation rate was found to have an error of $\sim 10 \%$.

50 The supersaturation in this manuscript is defined such that $\mathrm{S}=\mathrm{c} / \mathrm{c}_{\mathrm{o}}$ where $\mathrm{c}$ is the concentration of the ion and $\mathrm{c}_{\mathrm{o}}$ is the equilibrium solubility concentration. When the ions forming the crystallizing salt are present at $1: 1$ ratios and the activity of the ions is $\sim 1$, this is reasonably accurate. This approximation has been made because we do not know the extent of 55 barium ion complexation with the calixarene molecules in question. The $\%$ inhibition is also calculated based on the following normalized equation:

$$
\text { \%Inhibition }=100 *\left[\left(\mathrm{k}_{0}-\mathrm{k}\right) / \mathrm{k}_{0}\right]
$$

where $\mathrm{k}_{0}$ is the de-supersaturation rate, for the control run (absence of 60 impurity) and $\mathrm{k}$ is the de-supersaturation rate, for the experiment with impurity present.

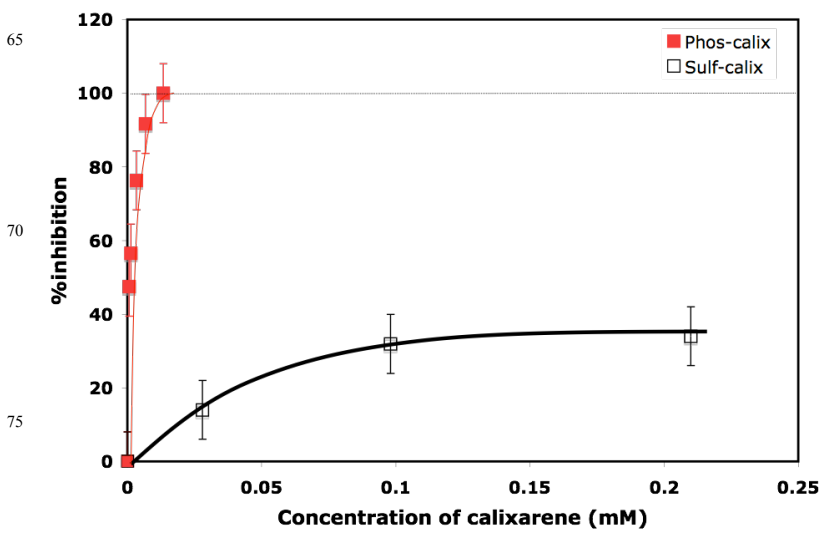

Fig. S1 \%inhibition of barium sulfate crystallization derived from conductivity experiments for the two calixarene 80 molecules. Filled squares correspond to the $p$ phosphonatocalix[4]arene and open squares correspond to the $p$ sulfonatocalix [4]arene

\section{Morphology of particles}

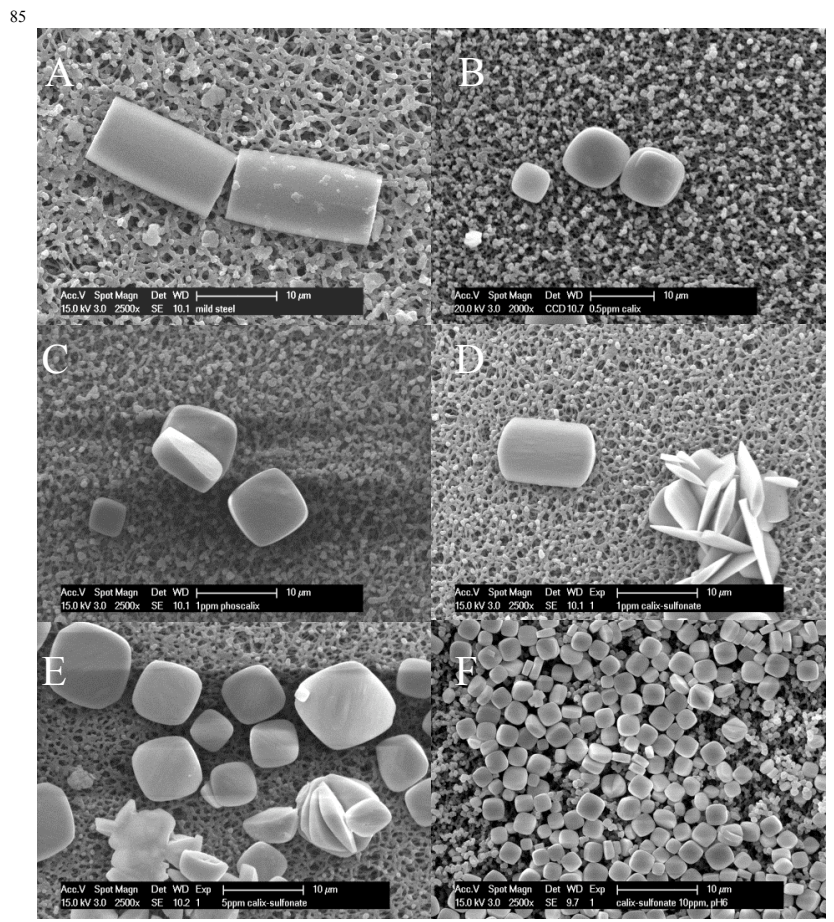

Fig S2. SEM images of barium sulfate formed in the presence of 90 phosphonated calixarene 2 A) $0 \mathrm{mM}$ B) $0.0007 \mathrm{mM}$ and C) 0.0013 $\mathrm{mM}$ and in the presence of sulfonated calixarene $1 \mathrm{D}) 0.0015 \mathrm{mM} \mathrm{E}$ ) $0.0073 \mathrm{mM}$ and F) $0.0145 \mathrm{mM}$ 

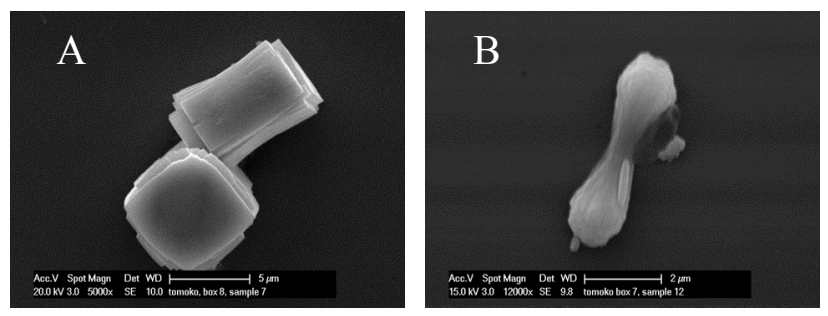

Fig S3. Barium sulfate particles formed in the presence of A) 0.015 $\mathrm{mM}$, and B) $0.030 \mathrm{mM}$ p-sulfonatocalix[4] arene (A was conducted at a lower barium sulfate concentration of $0.05 \mathrm{mM}$ while $B$ was at 0.25 $5 \mathrm{mM} \mathrm{BaCl}_{2}$ and $\mathrm{Na}_{2} \mathrm{SO}_{4}$ )

\section{SAED}
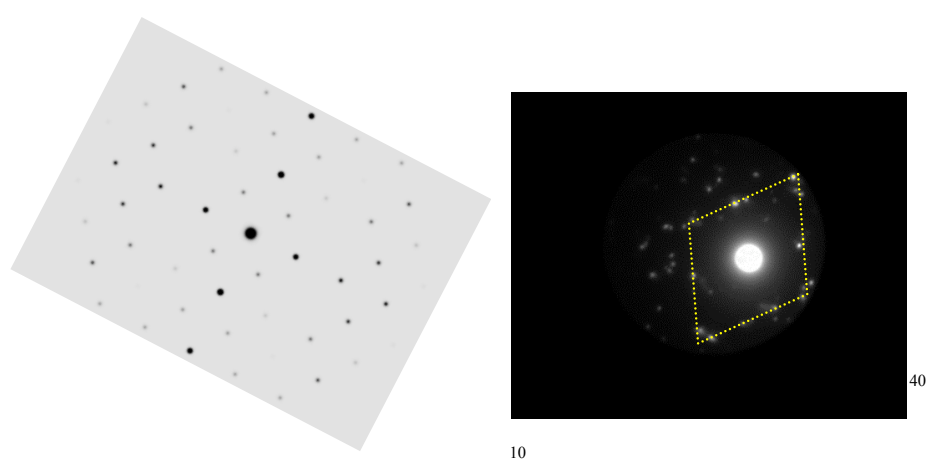

Fig. S4 Predicted SAED for the (100) zone (using Crystal Diffract $\left.^{\circledR}\right)$ and actual SAED showing the alignment is consistent with the (100) zone$$
15
$$

\section{Simulated AFM image}

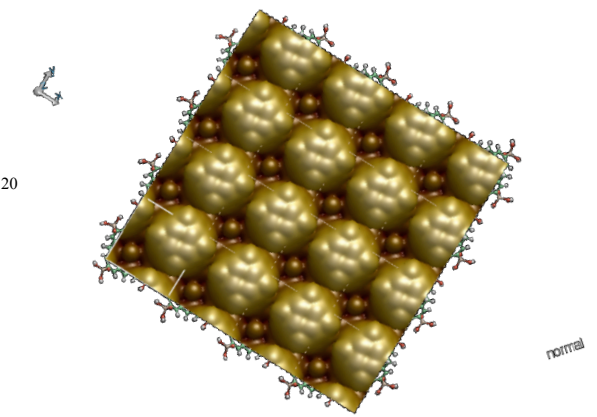

${ }_{25}$ Fig. S5 Simulated AFM image of $p$-phosphonato calix[4]arene bilayer viewed down the $c$-axis

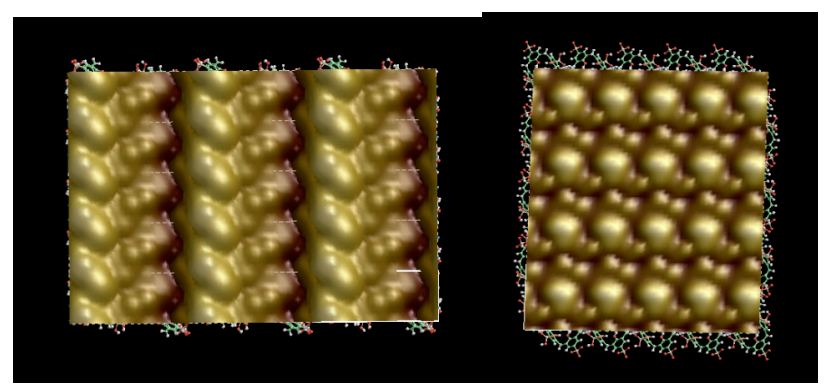

${ }_{35}(001)$ form 2

(010) form 2

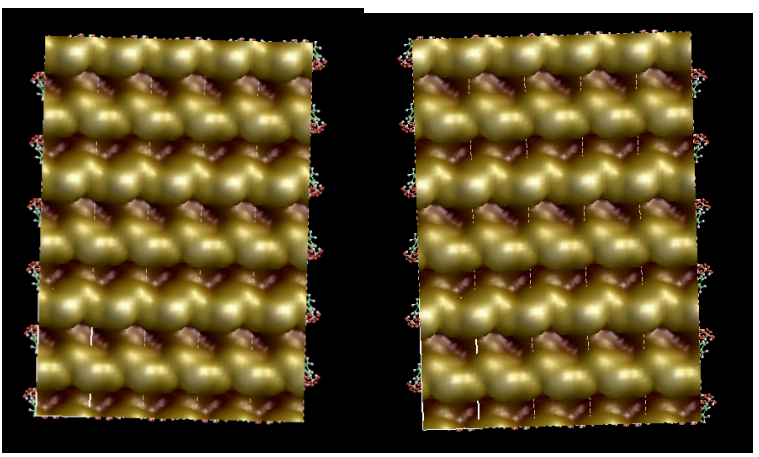

(010) form 1

(100) form 1

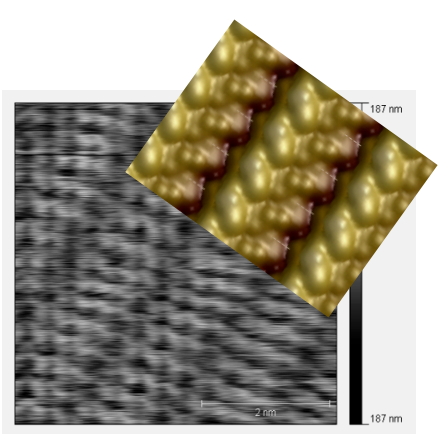

Fig. S6 Simulated AFM images of calcium complexes of $p$ phosphonato calix [4]arene

\section{References}

[S1] L. Ling, Y. Alias, A. N. Sobolev, C. L. Raston Crystal Growth and Design 20099 4497-4503.

[S2] A. D. Martin, A. N. Sobolev, M. A. Spackman, C. L. Raston, Crystal Growth \& Design 20099 3759-3764.

[S3] F. Jones, J. Clegg, A. Oliviera, A. L. Rohl, M. I. Ogden, G. M. Parkinson, A. M. Fogg, M. M. Reyhani (2001), CrystEngComm 40 1.

[S4] S. R. Freeman, F. Jones, M. I. Ogden, A. Oliviera, W. R. Richmond (2006), Crystal Growth \& Design 62579.

[S5] F. Jones, W. R. Richmond, A. L. Rohl (2006), J. Phys. Chem. B 1107414. 